\title{
Low Energy 500 eV Focused Argon Ion Beam Provided by Multi-Ions Species Plasma FIB for Material Science Sample Preparations
}

Chengge Jiao ${ }^{1}$, Jeremy Graham ${ }^{2}, \mathrm{Xu} \mathrm{Xu}^{3}$, Timothy Burnett ${ }^{3}$ and Brandon van Leer ${ }^{4}$

${ }^{1}$ Thermo Fisher Scientific, Eindhoven, Netherlands, ${ }^{2}$ Thermo Fisher scientific, Hillsboro, Oregon, United

States, ${ }^{3}$ The University of Manchester, Manchester, United Kingdom, ${ }^{4}$ Thermo Fisher Scientific, Hillsboro, Oregon, United States

Multi-Ions Species $\left(\mathrm{Xe}^{+}, \mathrm{O}^{+}, \mathrm{Ar}^{+}, \mathrm{N}^{+}\right.$) Plasma Focused Ion Beam (MIS-PFIB) microscope opens wider applications for sample preparation to conventional Gallium-Focused Ion Beam (G-FIB) microscopy world. The plasma ion source is a RF plasma generator utilizing external antenna inductively coupled plasma (ICP) source [1], and inside the source is an empty chamber, from where high angular intensity ion beam is extracted. Subsequent ion beam is then demagnified, collimated, scanned and focused on the sample. Switching between type of ions takes less than 10 mins automatically.

$\mathrm{Xe}^{+}$ion beam has being greatly accepted by FIB microscopy community providing the advantages of not only for high sputtering yield, high current milling at $30 \mathrm{keV}$ for a large 3D volume data acquisition and for correlated microscopy applications [2], but also for a much less ion implantation and amorphization of gallium free TEM specimen preparation [3]. Reactive oxygen ion beam dramatically improves sample milling quality for carbon-carbon bonding hard or soft materials by smoothing the cut-face very effectively during sputtering [4]. Low energy broad $\mathrm{Ar}^{+}$ion beam for sample preparation have been widely used for material science sample preparation for decades but it is not integrated with FIB-SEM DualBeam microscope in general.

In this paper, we focus on applying of a low energy to $500 \mathrm{eV} \mathrm{Ar}^{+}$focused ion beam provided by a MISPFIB/SEM DualBeam microscope for sample preparations, i.e., bulk sample surface cleaning for a builtin monochromated SEM BSE imaging in nano-scales and $\mathrm{Ar}^{+}$ion beam low energy $\mathrm{TEM}$ specimen final milling with built-in retractable STEM for real-time monitoring (RTM).

The materials used for $\mathrm{Ar}^{+}$ion beam bulk sample preparation is Al-Li-Cu-Mg alloy. $\mathrm{T}_{1}\left(\mathrm{Al}_{2} \mathrm{CuLi}\right)$ phase is the most prominent precipitates in artificially aged $\mathrm{Al}-\mathrm{Li}-\mathrm{Cu}-\mathrm{Mg}$ alloy with a $\mathrm{Li}$ content at about 1.4 - 1.5 wt.\% when the $\mathrm{Cu}$ : $\mathrm{Mg}$ ratio is higher than 3:1. To observe $\mathrm{T}_{1}$ phase distributions, it has been required to prepare a TEM specimen and observed it by a TEM/STEM. However, in this report, we observed $\mathrm{T}_{1}$ phases located both at grain boundary and inside the grain in BSE signal by a monochromated SEM at $3 \mathrm{keV}$. This is greatly assisted by the $500 \mathrm{eV} \mathrm{Ar}^{+}$ion beam cleaned sample surface in-situ in the MIS-PFIB/SEM microscope chamber. Al-Li alloys surface is very sensitivity to surface oxidation, $\mathrm{Ar}^{+}$ ion beam cleaning process removed not only the surface oxide contaminations but also carbon induced by electron beam scan, allowing epeated image acquisitions at the same location. The results are shown in figure 1 and 2. SEM images were taken automatically in monochromated beam condition with tiling and stitching for the whole $\mathrm{Ar}^{+}$cleaned area. The technique used for $\mathrm{Ar}^{+}$ion beam cleaning was deliberately set the beam to about $300 \mu \mathrm{m}$ defocus and scanned pixel overlapping to $98 \%$ with the pixel dwell time as short as $50-100 \mathrm{~ns}$. Such an approach for the sample cleaning results in not only removed surface 
contamination (oxidations and deposited carbon), but also smoothed the sample surface further even the beam is under the perpendicular incident to the sample surface.

TEM results acquired from $500 \mathrm{eV}$ final cleaned $\mathrm{CuO} / \mathrm{AL}$ interface TEM specimen are shown in figure 3 and 4. TEM specimens preparation could be optimized by using different ions at different sample preparation steps by switching types of ions freely, for example, $\mathrm{Xe}^{+}$beam protection layer can be deposited at $12 \mathrm{keV}$ by MultiChem $\mathrm{C}+\mathrm{Pt}$ gas mixing, and $\mathrm{O}^{+}$ion can be switched for rough milling such as diamond, then $\mathrm{Xe}^{+}$milling for cleaning and finally switch to low $\mathrm{keV} \mathrm{Ar}^{+}$for the last step cleaning . All process can be carried out by one system, Hydra PFIB. PFIB can prepare TEM specimen successfully with the key factors of a proper protection cap layer and the use of STEM for RTM. Ar ${ }^{+}$ion beam at low $\mathrm{kVs}(5 \mathrm{keV}$ to $500 \mathrm{eV}$ ) provides a much gentle milling allows the process in a controlled way.
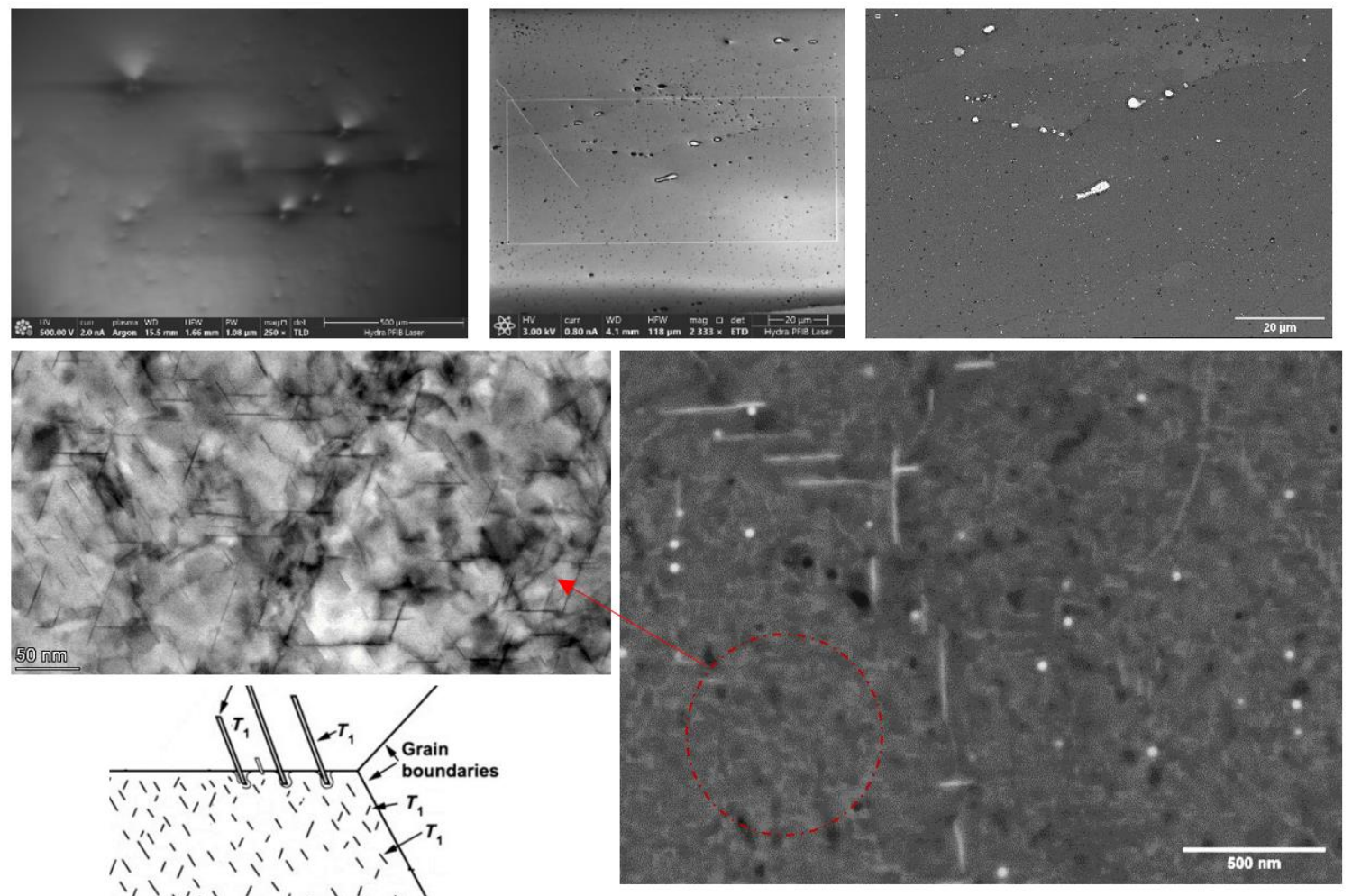

Figure 1. (Top left) $500 \mathrm{eV}$ Ar ion beam image acquired from a contaminated Al-Li alloy surface. (Top center) SEM secondary image after $500 \mathrm{eV}$ Ar ion beam clean for 30 minutes. The rectangle marker, 100 $\mu \mathrm{m}$ by $85 \mu \mathrm{m}$, was milled by Ar ion beam at $30 \mathrm{kV}$ at $60 \mathrm{pA}$, the line width is $\sim 150 \mathrm{~nm}$. (Top right) A tile and stitched $3 \mathrm{keV}$ SEM BSE images acquired by 437 tiles with a pixel size of $2.44 \mathrm{~nm} /$ pixel from 500 eV cleaned Al-Li alloy surface, the large white phases and smaller white dots are the intermetallic phases including $\mathrm{Al} 7 \mathrm{Cu} 2(\mathrm{Fe}, \mathrm{Mn}$ ) and $\mathrm{Al} 20 \mathrm{Cu} 2 \mathrm{Mn} 3$. (bottom left) $\mathrm{T} 1$ phase in a Al-Li alloy is visible not only the large plate-shape at grain boundary but also small needle-shape inside the grain, the width of the large T1-plate is about 12 - $15 \mathrm{~nm}$ measured by line scan FWHM. The small white dot phase is A120Cu2Mn3 dispersions. (Bottom right upper) TEM/STEM BF image from a plan view specimen prepared by low $\mathrm{kVs}$ Ar+ ion milling illustrating the $\mathrm{T} 1$ phase inside a grain. (bottom right lower) a diagram modified from reference [5] illustrates T1 at G.B and inside grain. The STEM image was acquired by Thermo Scientific Talos F200X TEM at $200 \mathrm{kV}$. 

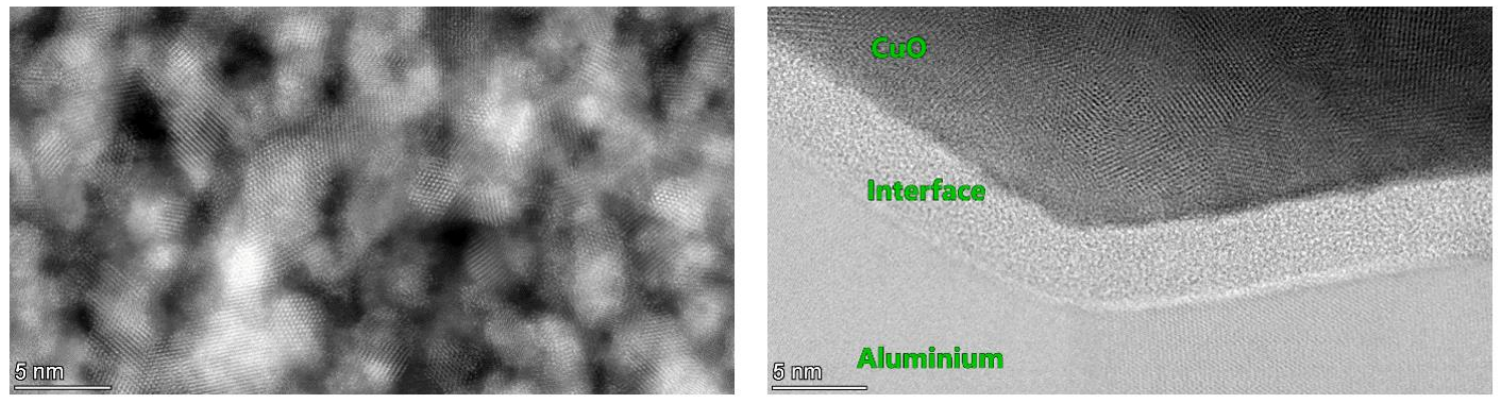

Figure 2. (left) STEM HAADF image shows Pt lattice image in protection cap layer deposited by $12 \mathrm{keV}$ $\mathrm{Xe}+$ ion beam with MultiChem GIS gas mixing Pt+C. (right) STEM BF image shows the interface between $\mathrm{CuO}$ and Aluminum. The specimen is gallium free with a final Ar+ ion beam "washed" at 500 $\mathrm{eV}$ while under SEM/STEM RTM. Images were acquired from Thermo Scientific Spectra 300 TEM at $300 \mathrm{kV}$, image pixel size: $19 \mathrm{pm} /$ pixel, Beam convergent angle: $30.0 \mathrm{mrad}$, current: $100 \mathrm{pA}$

References

[1] N. S. Smith, W. P. Skoczylas, S. M. Kellogg, D. E. Kinion, and P. P. Tesch, O. Sutherland, A. Aanesland, and R. W. Boswell, High brightness inductively coupled plasma source for high current focused ion beam applications, J. Vac. Sci. Technol. B, Vol. 24, No. 6, Nov/Dec (2006), 2902-2906

[2] Bartłomiej Winiarski, Grzegorz Pyka, Ali Chirazi, Multiscale Correlative Tomography Provides Critical Materials Characterization of Biomedical Implants, Microscopy and Analysis 31(6), December 2017, S4-S9.

[3] Ian MacLaren, Magnus Nord, Chengge Jiao, Emrah Yucelen, Liftout of High-Quality Thin Sections of a Perovskite Oxide Thin Film Using a Xenon Plasma Focused Ion Beam Microscope, , Microscopy and Microanalysis, 25(1), January 2019, 1-4, http://dx.doi.org/10.1017/S1431927618016239

[4] Valerie Brogden ,Cameron Johnson ,Chad Rue ,Jeremy Graham, Kurt Langworthy ,Stephen Golledge, and Ben McMorran, Material Sputtering with a Multi-Ion Species Plasma Focused Ion Beam, Advances in Materials Science and Engineering, Volume 2021, Article ID 8842777, https://doi.org/10.1155/2021/8842777

[5] Roberto J. Rioja and John Liu, The Evolution of Al-Li Base Products for Aerospace and Space Applications, Metallurgical and Materials Transactions A, Vol. 43A, September 2012, 3325-3337 